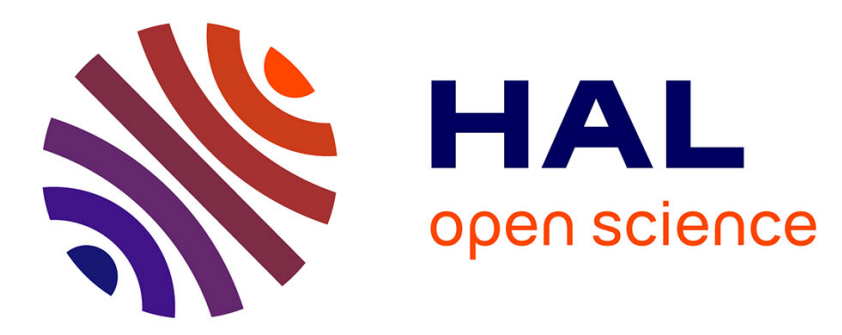

\title{
Good vibrations -Transition in drop generation from an immersed capillary tube
}

\author{
A. Bertrandias, H. Duval, Joel Casalinho, M. L. Giorgi
}

\section{To cite this version:}

A. Bertrandias, H. Duval, Joel Casalinho, M. L. Giorgi. Good vibrations -Transition in drop generation from an immersed capillary tube. EPL - Europhysics Letters, 2015, 111 (4), 10.1209/02955075/111/44004 . hal-01239101

\section{HAL Id: hal-01239101 \\ https://hal-centralesupelec.archives-ouvertes.fr/hal-01239101}

Submitted on 29 Nov 2016

HAL is a multi-disciplinary open access archive for the deposit and dissemination of scientific research documents, whether they are published or not. The documents may come from teaching and research institutions in France or abroad, or from public or private research centers.
L'archive ouverte pluridisciplinaire HAL, est destinée au dépôt et à la diffusion de documents scientifiques de niveau recherche, publiés ou non, émanant des établissements d'enseignement et de recherche français ou étrangers, des laboratoires publics ou privés. 
This document corresponds to the article published in EPL, of reference EPL, 111 (2015) 44004 (doi: 10.1209/0295-5075/111/44004). According to the copyright agreement, we have posted this version of the accepted manuscript on the institution website repository, as a file not formatted by EPLA. The link to the online EPL abstract is:

http://epljournal.edpsciences.org/articles/epl/abs/2015/16/epl17314/epl17314.html

An erratum was published, of reference EPL, 114 (2016) 59901 (doi: 10.1209/0295-5075/114/59901). The link to the erratum page is:

http://iopscience.iop.org/article/10.1209/0295-5075/114/59901

\title{
Good vibrations - Transition in drop generation from an immersed capillary tube
}

\author{
A. Bertrandias ${ }^{(a)}$, H. Duval ${ }^{(b)}$, J. Casalinho and M. L. Giorgi \\ Grande voie des vignes, 92295 Châtenay-Malabry, France \\ published online 4 September 2015 \\ PACS 47.55.db - Drop and bubble formation \\ PACS 47.55.df - Breakup and coalescence \\ PACS 47.35.Pq - Capillary waves
}

Laboratoire de Génie des Procédés et Matériaux (LGPM), CentraleSupélec, Université Paris Saclay -

received 23 June 2015; accepted in final form 9 August 2015

\begin{abstract}
We experimentally investigate single drop formation from a vibrating capillary tube immersed in a second immiscible liquid. At set forcing frequencies, significantly smaller drops are generated when a threshold amplitude is reached. We show that a drop grows and resonates in first mode once the drop resonance frequency and the forcing frequency coincide. The drop then detaches by a stretching mode if its elongation ratio exceeds a critical value, function of the drop to pore diameter ratio. The detached drop diameter is well described by the Bisch et al. expression for bound drop resonance frequency. Otherwise, the drop grows, leaves the first-mode resonance range and detaches at a larger size by dripping. The threshold and drop diameters are well predicted by a simple LFHO-based model.
\end{abstract}

Introduction. - Controlling drop breakup is key for industries performing emulsification such as the food, pharmaceutical and chemical industries. Indeed, drop size and distribution affect emulsion rheological properties and stability. Membrane emulsification (ME) consists in forcing a dispersed phase through a porous membrane. The drops are directly generated on the other side of the membrane into a continuous flowing phase, which exerts shear on the drops [1]. ME is commonly operated in dripping (drop by drop) mode.

${ }^{\text {(a) }}$ E-mail: aude.bertrandias@centralesupelec.fr

(b) E-mail: herve.duval@centralesupelec.fr 
In recent years, a few attempts were made to couple ME with vibration to better control drop detachment, thus drop size [2-4]. Most investigations concern longitudinal vibrations (see [2] and references therein). In these studies, the shear stress experienced by the drop is mainly due to the membrane oscillation and drop size is controlled by the peak shear stress at the membrane surface. The drop size does not depend on the membrane excitation frequency apart from its influence on shear stress [2]. ME coupled with transversal vibrations (perpendicular to the membrane surface) has been less studied [3,4]. In this case, membrane vibration induces axial vibrations of the drops. Arnaud found a strong decrease in drop size when the membrane (mean pore size of $0.8 \mu \mathrm{m}$ ) was excited at a frequency of $15 \mathrm{kHz}$ (unknown forcing amplitude). The peak of the volume-weighted drop size distribution was shifted from $30 \mu \mathrm{m}$ without vibration to $10 \mu \mathrm{m}$ with vibration [3]. The mechanisms at hand were not explained $[3,4]$.

However, axial oscillations of bound drops undergoing forced vibrations have been extensively investigated since the pioneering experimental work of Bisch et al. [5]. Strani and Sabetta examined the case of small linearised oscillations and found an additional vibration mode $(n=1)$ for the bound drop, associated with the translation motion of the centre of mass of the drop [6]. It is reminded that the $n=2$ mode is the first vibration mode for a free drop as established by Lamb [7]. The response of drops undergoing large-amplitude vibrations has been studied by DePaoli et al. and Wilkes and Basaran [8,9]. They showed that bound drops behave as soft nonlinear oscillators and can exhibit hysteretic responses. Finally, Wilkes and Basaran and Kim studied drop detachment in air under strong forcing [10,11]. Wilkes and Basaran examined drop ejection from an oscillating rod using computational fluid dynamics. They found that above a critical forcing amplitude, a pendant drop (with a pinned contact line) breaks up and a primary drop is ejected [10]. Kim experimentally studied the fall-off of a pendant drop (with a free contact line) from a smooth solid vibrating surface. Kim found that the vibration amplitude inducing fall-off is minimal when the forcing frequency and resonance frequency of the drop coincide [11].

In order to understand the effect of vibration on drop detachment in ME, we focus on a simplified configuration where rising drops form from an axially vibrating capillary tube into an unbounded liquid under gravity. A dedicated transparent setup has been designed to observe drop formation. Contrarily to cross-flow ME, there is no continuous phase flow and the membrane reduces to a single pore, i.e., the capillary tube, so potential interactions between drops at adjacent pores are avoided. Drops are constantly fed dispersed phase, thus their size increases with time whereas in the studies on drop oscillation mentioned above, the drop size is set. The aim of the present paper is to study drop generation assisted by axial oscillations, so as to identify the different regimes and the mechanisms involved depending on the excitation parameters.

Experimental setup. - Oil-in-water emulsions were formed with dodecane (99\%, viscosity $\eta_{d}=1.34$ $\mathrm{mPa}$.s at $25^{\circ} \mathrm{C}$, density $\rho_{d}=750 \mathrm{~kg} \cdot \mathrm{m}^{-3}$ at $25^{\circ} \mathrm{C}$ ) as the dispersed phase and distilled water (viscosity $\eta_{c}=0.89 \mathrm{mPa} . \mathrm{s}$ at $25^{\circ} \mathrm{C}$, density $\rho_{c}=997 \mathrm{~kg} \cdot \mathrm{m}^{-3}$ at $25^{\circ} \mathrm{C}$ ) as the continuous, aqueous phase. The interfacial tension was of $\gamma=46.0 \pm 2.1 \mathrm{mN}^{-1}$ as measured by the rising drop method with a tensiometer (Tracker, I.T. Concept, Teclis), averaged between $10 \mathrm{~s}$ and $20 \mathrm{~s}$ after drop formation.

A single-pore vibrating setup has been designed to observe drop formation (fig. 1). The setup consists in a transparent tank filled with $70 \mathrm{~mL}$ of stationary aqueous phase, in which a glass capillary 
tube is placed. The dispersed phase is fed through the capillary with a syringe pump (R-99, Razel Instruments). For the pore diameter $D_{p}=0.32 \mathrm{~mm}$, three dispersed phase flow rates are tested: $q=$ $2.5 \mu \mathrm{L} . \mathrm{s}^{-1}, 4.3 \mu \mathrm{L} . \mathrm{s}^{-1}$ and $6.5 \mu \mathrm{L} . \mathrm{s}^{-1}$. When the influence of $D_{p}$ is investigated, dispersed phase flow rates are set at $q=2.2 \mu \mathrm{L} . s^{-1}, 6.5 \mu \mathrm{L} . \mathrm{s}^{-1}, 6.9 \mu \mathrm{L} . \mathrm{s}^{-1}$ and $10.1 \mu \mathrm{L} . \mathrm{s}^{-1}$ (accuracy within $2 \%$ ) for $D_{p}=$ $0.11 \mathrm{~mm}, 0.32 \mathrm{~mm}, 0.35 \mathrm{~mm}$ and $0.75 \mathrm{~mm}$ respectively. The flow in the capillary is laminar as the associated Reynolds numbers lie between $\operatorname{Re}_{\text {cap }}=5$ and $\operatorname{Re}_{\text {cap }}=22$ (with $\operatorname{Re}_{\text {cap }}=\rho_{d} v_{d} D_{p} / \eta_{d}$ and $v_{d}=4 q /\left(\pi D_{p}^{2}\right)$, the dispersed phase velocity).

The capillary is fixed on a vibrating exciter (Bruel \& Kjaer 4810) and is driven with a sinusoidal motion function of time: $x=(1 / 2) A_{p p} \sin (2 \pi f t)$. The vibration is parallel to the capillary tube axis, so drops undergo axial vibrations. Forcing frequencies are $f=30 \mathrm{~Hz}$ to $150 \mathrm{~Hz}(10 \mathrm{~Hz}$ intervals until $100 \mathrm{~Hz}$, variable intervals above depending on $D_{p}$ ). Peak-to-peak amplitudes range from $A_{p p}=0 \mathrm{~mm}$ to $2 \mathrm{~mm}$, measured by an LED sensor (M5L/2, Bullier International), with an uncertainty in the order of $10 \mu \mathrm{m}$.

A halogen cold light illuminates the system. A high-speed camera (Phantom v310) mounted with a macro lens (AF Zoom-Micro Nikkor 70-180mm f/4.5-5.6D ED, Nikon) captures drop formation images with a resolution of $800 \times 600$ pixels. Acquisition frequency is set at 10 times the forcing frequency and at $100 \mathrm{fps}$ for trials without vibration. The images are analysed using ImageJ with the scale set by the capillary outside diameter. Data are provided including average drop diameters $D_{d}$ and axial drop elongations. The maximum relative errors are of $7 \%$, mainly due to statistical dispersion, as the error due to image resolution is of $1 \%$. Experiments were carried out in triplicate and the error bars in the figures correspond to statistical dispersion.

After experimentation, the setup is cleaned with a surfactants solution (3 vol\%, Mucasol, Merz) and thoroughly rinsed with distilled water. As a result, the glass surface is hydrophilic and the capillary outside diameter does not influence dodecane drop detachment.

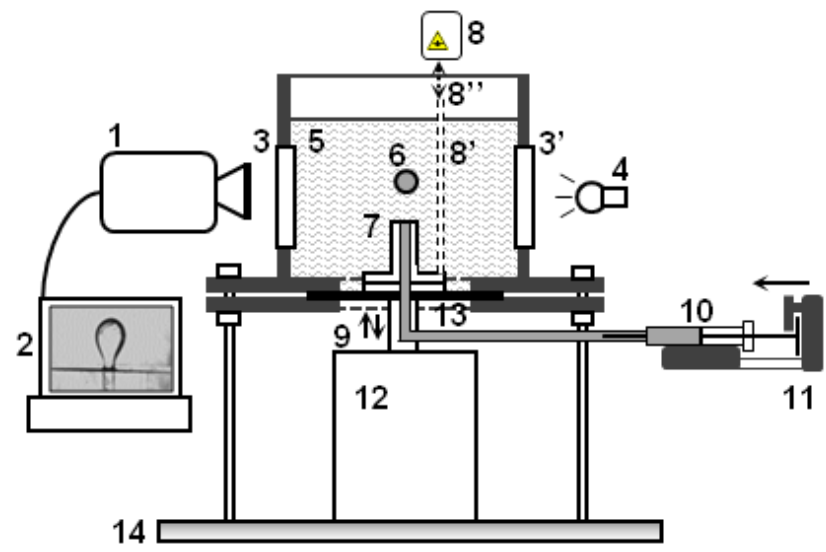

Figure 1: Experimental setup: 1, high speed camera; 2, computer; 3 and 3', windows; 4, light source; 5, continuous phase; 6 , dispersed phase; 7, glass capillary; 8, 8' and 8', LED sensor, emerging rod and distance measured; 9 , vibrating motion; 10 , syringe; 11 , syringe pump; 12 , exciter; 13 , flexible seal; 14, optical table.

Drop detachment mode without vibration. - Without vibration, experiments are in dripping mode for the dispersed phase flow rates and pore diameters tested. Indeed, a drop by drop formation is observed, with the drops formed close to the capillary tip. 
In dripping mode, detachment occurs when buoyancy $F_{b}$ exceeds the maximum capillary force $F_{\gamma}^{\max }=\pi D_{p} \gamma$ that the drop neck can withstand without breaking, as stated by Tate's law [12]:

$$
F_{b}=\mathcal{F}_{H B} F_{\gamma}^{\max } \text {, i.e. }\left(\rho_{c}-\rho_{d}\right) \frac{\pi}{6} D_{d}^{3} g=\mathcal{F}_{H B} \pi D_{p} \gamma
$$

with $\mathcal{F}_{H B}$ the Harkins Brown correction factor, introduced to account for the fraction of liquid volume which remains attached to the capillary tip after drop break off [13].

Theoretical drop diameters were calculated from eq. (1) using the factor $\mathcal{F}_{H B}$ proposed by Mori [14]. They were compared to those measured. The relative differences between calculated and measured diameters vary between $-7 \%$ and $5 \%$ depending on pore diameters and dispersed phase flow rates. It is in the order of drop diameter uncertainty.

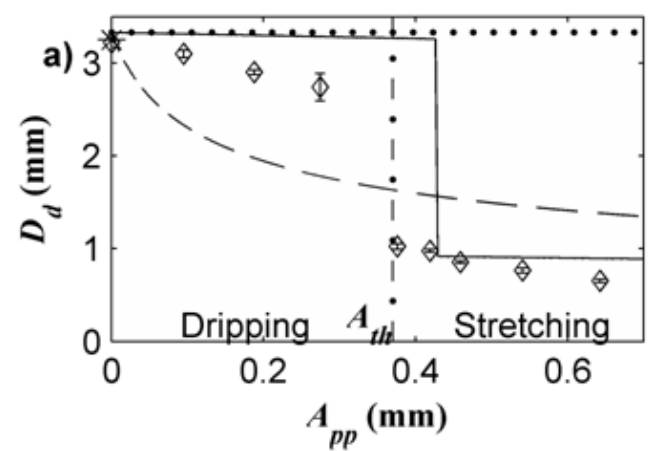

b)
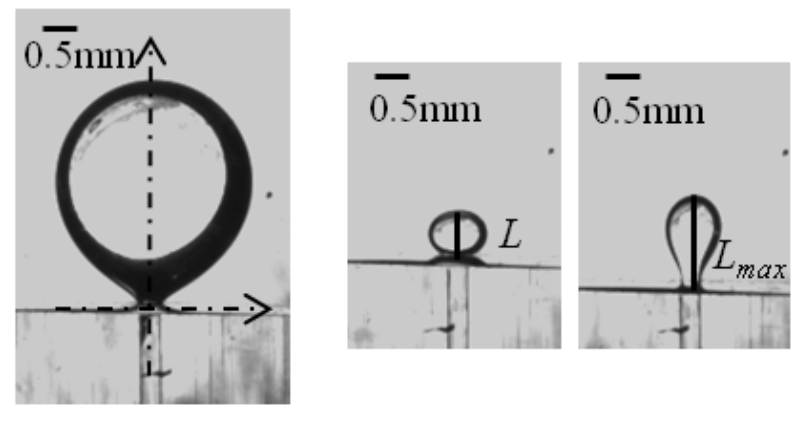

Figure 2: (a) Drop diameter $D_{d}$ depending on the peak-to-peak capillary amplitude $A_{p p}$ for $f=100 \mathrm{~Hz}, D_{p}=$ $0.32 \mathrm{~mm}, q=4.3 \mu \mathrm{L} \cdot \mathrm{s}^{-1}:(\diamond)$ measurements, measured amplitude threshold (dash-dotted line), $\left(^{*}\right)$ estimates from eq. (1), estimates from the balance $F_{b}=F_{\gamma}^{\max }$ (dotted line), estimates from the balance $F_{b}+F_{\text {exc }}^{\text {peak }}=F_{\gamma}^{\text {max }}$ (dashed line), estimates from eq. (9) (solid line). (b) Drop image just before detachment in dripping mode (left, $A_{p p}=0.095 \mathrm{~mm}$ ), in stretching mode (centre and right, $A_{p p}=0.419 \mathrm{~mm}$ ).

Detachment modes with vibration. - Amplitude sweeps from low to high amplitudes were carried out at set forcing frequencies. For $f=30 \mathrm{~Hz}$ to $f=150 \mathrm{~Hz}$, two modes of detachment are observed, characterized by large or small drops. These modes are separated by a threshold amplitude $A_{t h}$ (fig. 2). For other frequencies, the transition could not be observed due to limitations of the vibrating exciter. This was also the case above $110 \mathrm{~Hz}$ and $120 \mathrm{~Hz}$ for $D_{p}=0.11 \mathrm{~mm}$ and $0.75 \mathrm{~mm}$ respectively.

For $A_{p p}<A_{t h}$, drops detach at large sizes, close to those for drops formed without vibration $\left(A_{p p}=0\right)$. As $A_{p p}$ increases, drop size decreases and an increasing deformation of the drop interface is seen. However drops still detach in dripping mode (fig. 2(b), left). The large apparent black ring inside the drop (fig. 2(b), left) is due to the differences in the refractive index of dodecane $\left(\mathrm{n}_{d}=1.42\right)$ and water $\left(\mathrm{n}_{c}=1.33\right)$.

For $A_{p p} \geq A_{t h}$, enhanced drop detachment occurs: smaller monodispersed drops are generated at a faster rate than in dripping mode. A decrease of $45 \%$ (for $f=30 \mathrm{~Hz}$ ) to $76 \%$ (for $f=150 \mathrm{~Hz}$ ) in drop diameter is observed at $A_{t h}$ compared to without vibration. Beyond the threshold, drop diameters continue to decrease with increasing $A_{p p}$. Drops are elongated at breakup in this mode (fig. 2(b), right). This was named the stretching mode. 
Threshold. - The higher the forcing frequency, the lower the threshold amplitude (fig. 3). The threshold amplitude roughly scales as $f^{-1}$ for $D_{p}=0.32 \mathrm{~mm}$ and $0.35 \mathrm{~mm}$. For $D_{p}=0.75 \mathrm{~mm}$, no scaling law emerges. For $D_{p}=0.11 \mathrm{~mm}$, the threshold amplitude is around twice higher compared to other pore diameters and scales as $f^{-2 / 3}$. Dispersed phase flux does not significantly affect $A_{t h}$ (not shown).

The diameters of the drops generated at $A_{t h}$ decrease with increasing forcing frequency (fig. 4). Furthermore, it appears that drop diameters scale as $f^{-1 / 2}$ for all pore diameters. Also, as expected, the larger the pore diameter, the larger the drops produced. Lastly, as the dispersed phase flux increases, a slight increase in drop diameter is observed (not shown). It is attributed to the extra dispersed phase fed to the drop during necking.

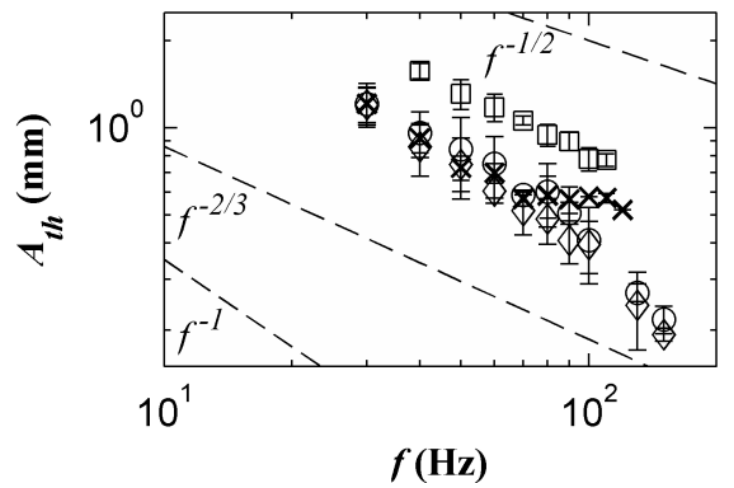

Figure 3: Threshold amplitude depending on the forcing frequency: () $D_{p}=0.11 \mathrm{~mm}, q=2.2 \mu \mathrm{L} \cdot \mathrm{s}^{-1}$, (৩) $D_{p}=0.32 \mathrm{~mm}, q=6.5 \mu \mathrm{L} . \mathrm{s}^{-1}$, (o) $D_{p}=0.35 \mathrm{~mm}, q=6.9 \mu \mathrm{L} . \mathrm{s}^{-1}$, (x) $D_{p}=0.75 \mathrm{~mm}, q=10.1 \mu \mathrm{L} . \mathrm{s}^{-1}$.

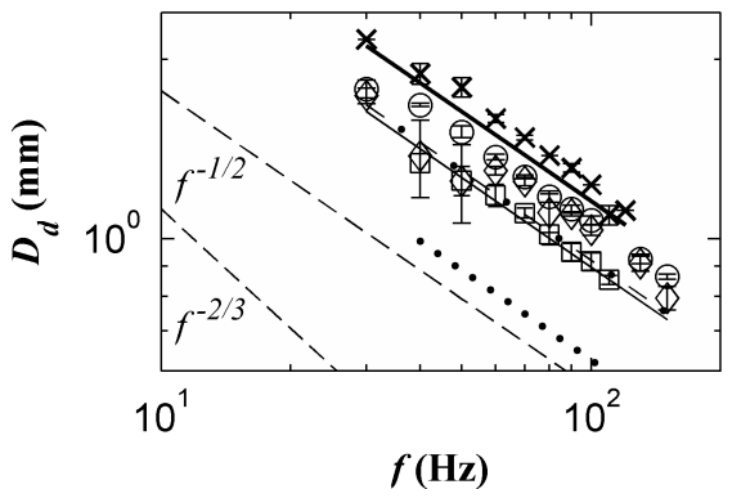

Figure 4: Drop diameter depending on the forcing frequency: (ㅁ) $D_{p}=0.11 \mathrm{~mm}, q=2.2 \mu \mathrm{L} . \mathrm{s}^{-1},(\diamond) D_{p}=0.32$ $\mathrm{mm}, q=6.5 \mu \mathrm{L} . \mathrm{s}^{-1}$, (o) $D_{p}=0.35 \mathrm{~mm}, q=6.9 \mu \mathrm{L} . \mathrm{s}^{-1}$, (x) $D_{p}=0.75 \mathrm{~mm}, q=10.1 \mu \mathrm{L} . \mathrm{s}^{-1}$. Estimates from eq. (9): $D_{p}=0.11 \mathrm{~mm}$ (dotted line), $D_{p}=0.32 \mathrm{~mm}$ (dash-dotted line), $D_{p}=0.35 \mathrm{~mm}$ (solid line), $D_{p}=0.75$ $\mathrm{mm}$ (thick solid line).

Resonance. - In the moving non-inertial frame of reference where the capillary is still (axes defined in fig. 2(b)), the forces exerted on the drop due to the capillary motion are the inertial force and the associated Archimedes' thrust. We note that, in the present setup, the continuous phase above the capillary and its support is accelerated by the exciter, as shown by Faraday waves at the free surface. Thus, the resulting excitation force reads

$$
F_{e x c}=\left(\rho_{c}-\rho_{d}\right) \frac{\pi}{6} D_{d}^{3} a_{c a p}
$$


with $a_{c a p}=(1 / 2) A_{p p} \omega^{2} \sin (\omega t+\pi)$ the capillary acceleration in the laboratory inertial frame and $\omega=2 \pi f$ the forcing pulsation. Hence, the peak excitation force is

$$
F_{\text {exc }}^{\text {peak }}=\left(\rho_{c}-\rho_{d}\right) \frac{\pi}{12} D_{d}^{3} \omega^{2} A_{p p}
$$

A Tate-like model based on the balance between the maximum driving force of drop detachment $\left(F_{b}+F_{\text {exc }}^{\text {peak }}\right)$ and the restoring capillary force $F_{\gamma}^{\text {max }}$ is represented in fig. $2(\mathrm{a}) . \mathcal{F}_{H B}$ is omitted as it is a first-order correction. This simple model does not account for the experimental results as the sudden jump in drop diameter is not predicted. Additionally, the measured drop diameters in stretching mode are significantly lower than the predicted diameters.

Drop generation footage reveals that as a drop grows, its length $L$ (measured from capillary tip to drop apex, fig. 2(b), centre) oscillates and it elongates more just before enhanced detachment. In addition, we found a strong correlation between drop diameter at $A_{t h}$ and forcing frequency (fig. 4). These elements suggest that drop resonance is involved in enhanced detachment.

Figure 5 presents typical variations of drop length $L$ with time $t$ (arbitrary time origin) for $A_{p p}<$ $A_{t h}$. These variations coincide with the drop apex motion in the capillary frame. Figure 5 simultaneously shows the displacement $x$ of the capillary tip in the laboratory inertial frame. We observe that mean drop length increases as dispersed phase is constantly fed to the drop. Drop length oscillates around the mean due to the excitation force $F_{\text {exc }}$. Drop oscillation amplitude is significantly lower than the capillary one. Indeed, the amplitude of the drop excitation force per mass unit is lower than the capillary acceleration amplitude, by a factor $\phi=\left(\rho_{c}-\rho_{d}\right) \rho_{d}=0.325$. From $t \cong 0.12 \mathrm{~s}$, the drop oscillation amplitude increases until a maximum reached at $t \cong 0.21 \mathrm{~s}$, then decreases. From $t \cong$ $0.12 \mathrm{~s}$ to $0.26 \mathrm{~s}$, drop oscillation amplitudes are higher than outside this area. This relates a resonance zone (RZ) of the drop. Resonance may be involved in stretching mode as drop sizes in the RZ are close to detached drop sizes at $A_{t h}$.

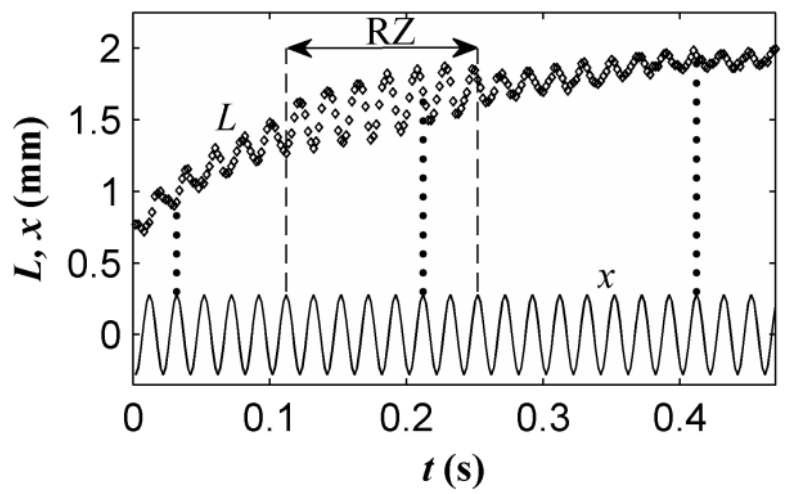

Figure 5: $(\diamond)$ Drop length $L$, capillary displacement $x$ (solid line), approximate boundaries of the resonance zone RZ (dashed line), phase shift estimation guidelines (dotted line): $f=50 \mathrm{~Hz}, D_{p}=$ $0.32 \mathrm{~mm}, A_{p p}=0.56 \mathrm{~mm}<A_{t h}=0.88 \mathrm{~mm}, q=4.3 \mu \mathrm{L} . \mathrm{s}^{-1}$.

Drop shapes in the resonance zone (fig. 2(b), centre and right) correspond to the shapes reported by Bisch et al. [5] for the $n=1$ vibration mode of bound drops. A simple empirical formula for mode 1 resonance frequency $f_{r}$ is given by Bisch et al. [5]: 


$$
f_{r}=\frac{1}{2 \pi} \sqrt{\frac{6 K \gamma}{\pi \rho_{d}}} \frac{\sqrt{D_{p}}}{D_{d}^{2}}
$$

with $K=9$ for any two immiscible fluids with equal densities. In our experiments, densities are close as $\rho_{c} / \rho_{d}=1.3$. Equation (4) was established for drop to pore diameter ratios of 1.3 to 7 . In our case, the ratios are of 1.3 to 12.3 . We note that the scaling law followed by the drop diameter at $A_{t h}$, i.e. $D_{d} \sim f^{-1 / 2}$, is consistent with eq. (4). In comparison, if a bound drop resonated exactly as a free drop, the drop diameter would scale as $f^{-2 / 3}[7]$.

From eq. (4), we calculate the resonance frequency of the drops detached at $A_{t h}$. The diameter of the resonating bound drop is assimilated to the detached drop diameter. These frequencies concur well with the experimental forcing frequencies (fig. 6). We conclude that once a drop reaches a critical size such that its resonance frequency and the forcing frequency coincide, it resonates in mode 1 and detaches if the capillary oscillation amplitude exceeds $A_{t h}$.

For $D_{p}=0.11 \mathrm{~mm}$, the calculated frequencies are lower than the forcing frequencies (fig. 6). Indeed, drop to pore diameter ratios range from 7.5 to 12.3 , which is above where eq. (4) was validated. The calculations of Strani and Sabetta [6] show that the bound drop first resonance frequency scales as $f_{r} \sim D_{d}^{\alpha}$ where $\alpha$ varies from -2.9 to -1.75 when the drop to pore diameter ratios vary from 1.3 to 7 , respectively. They show that eq. (4) underestimates the resonance frequency when $D_{d} / D_{p}>7$, which is consistent with the deviation seen for $D_{p}=0.11 \mathrm{~mm}$.

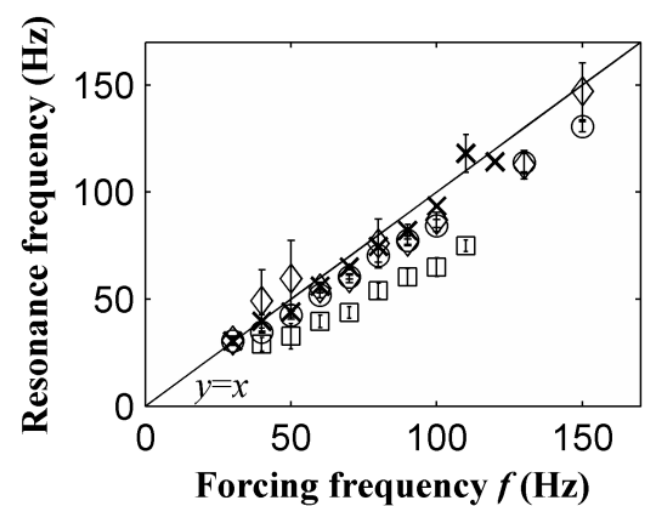

Figure 6: Calculated mode 1 resonance frequency [5] versus set experimental frequency: () $D_{p}=0.11 \mathrm{~mm}$, $q=2.2 \mu \mathrm{L} . \mathrm{s}^{-1},(\diamond) D_{p}=0.32 \mathrm{~mm}, q=6.5 \mu \mathrm{L} . \mathrm{s}^{-1}$, (o) $D_{p}=0.35 \mathrm{~mm}, q=6.9 \mu \mathrm{L} . \mathrm{s}^{-1}$, (x) $D_{p}=0.75 \mathrm{~mm}, q=$ $10.1 \mu \mathrm{L} \cdot \mathrm{s}^{-1}$.

We suppose that the resonating bound drop behaves as a linearly forced harmonic oscillator (LFHO) with moderate damping and that drop growth is slow enough to consider steady-state oscillations. Before the RZ, the drop is so small that its resonance frequency is notably greater than the forcing frequency $f$. In this case, the LFHO model [15] predicts that drop oscillations are in phase with the excitation force and that the drop oscillation amplitude is close to the forcing amplitude, i.e. $\phi A_{p p}$. This agrees with drop behavior (fig. 5). We remind that the capillary motion $x(t)$ is in phase opposition with the excitation force $F_{\text {exc }}(t)$ (eq. (2)). After the RZ, the drop is so large that its resonance frequency is notably lower than $f$. Then, the LFHO model [15] predicts that drop oscillations are in phase opposition with the excitation force and that the drop oscillation amplitude becomes small compared to the forcing amplitude when $f$ is large enough. This explains why the 
Tate-like model based on peak excitation force (dashed line, fig. 2(a)) rapidly underestimates drop diameters in dripping mode. At resonance, drop oscillation amplitudes are maximal and the phase shift is equal to $\delta=-\pi / 2$ (fig. 5), which is in accordance with the LFHO model [15].

In fig. 5, the RZ is wide as drop size increases slowly with the dispersed phase flow rates used: the drop diameter increase per oscillation is of about $1.3 \%$ in the RZ.

When the drop does not detach in the RZ, it grows and detaches at a larger size in dripping mode, at $2.36 \mathrm{~s}$ in the example in fig. 5 (not shown). At this point, drop oscillations (which became weak) increase again, but with a lesser amplitude than in the RZ. This is attributed to the excitation of a higher resonance mode of the drop. This explains why in the dripping mode, drop diameter steadily decreases as $A_{p p}$ increases.

Force-based analysis. - To account for the transition from dripping to stretching mode, we propose a model where the drop is described as a LFHO and the condition for drop detachment is expressed with a force-based criterion. The system is certainly nonlinear since oscillations can be large enough to induce drop detachment $[8,9]$. However, it is expected that the main features of the transition can be understood from this approximation.

The bound drop is subjected to a restoring capillary force, made up of a stationary and oscillatory part. The stationary part balances the buoyancy force and can be expressed as

$$
F_{\gamma}^{\text {stat }}=-\frac{\pi}{6} D_{d}^{3}\left(\rho_{c}-\rho_{d}\right) g
$$

The oscillatory part responds to the excitation force. According to the LFHO model, this part reads

$$
F_{\gamma}^{o s c}=-\frac{\pi}{6} D_{d}^{3} \rho_{d} \omega_{1}^{2} x_{d}(t)
$$

with $\omega_{1}$ the mode 1 eigenpulsation of the bound drop without damping and $x_{d}(t)$ the displacement of the centre of mass of the drop. We assume that the buoyancy force negligibly affects the drop eigenpulsation and damping constant. Indeed, the drop Bond number, $\mathrm{Bo}_{d}=\left(\rho_{c}-\rho_{d}\right) g\left(D_{d} / 2\right)^{2} / \gamma$, in the RZ is significantly lower than 1 . Assuming quasi-steady-state oscillations, the drop centre-ofmass motion is given by $x_{d}(t)=(1 / 2) A_{d}(\omega) \sin (\omega t+\delta)$ with the peak-to-peak amplitude

$$
A_{d}(\omega)=-\frac{\omega^{2} \phi A_{p p}}{\sqrt{\left(\omega_{1}^{2}-\omega^{2}\right)^{2}+4 \beta^{2} \omega^{2}}}
$$

where $\omega_{1}$ is related to $\omega_{r}$ (resonance pulsation) by the expression $\omega_{r}^{2}=\omega_{1}^{2}-2 \beta^{2}$, with $\omega_{r}=2 \pi f_{r}$ ( $f_{r}$ the resonance frequency) and $\beta$ the damping constant. For moderate damping, $\omega_{r} \cong \omega_{1}$ and the peak-to-peak amplitude of the steady-state oscillations at resonance is given by $A_{d}^{r} \cong Q\left(\phi A_{p p}\right)$ with the quality factor $Q=\omega_{1} /(2 \beta)$.

We first estimated $Q$ from eq. (8) given by Bisch et al. [5], which relates $\beta$ to the resonance frequency $f_{r}$ and to the phase properties (valid for $\eta_{d} / \rho_{d}>\eta_{c} / \rho_{c}$ as is our case):

$$
\beta / f_{r}=4.5 \times 10^{3}\left(\eta_{d} / \rho_{d}\right)+2.32 \times 10^{5}\left(\eta_{c} / \rho_{c}\right)
$$

We found $Q=14.6$. On the other hand, at set $f$, we measured the maximum amplitude $A_{d}^{\max }$ of the drop centre-of-mass motion in the RZ (for $\left.A_{p p}<A_{t h}\right)$. We estimated Q by $A_{d}^{\max } /\left(\phi A_{p p}\right)$ and 
found significantly lower values than from eq. (8). For example, in the conditions of fig. 2, $A_{d}^{\max } /\left(\phi A_{p p}\right)=4.5$. This ratio is independent of $A_{p p}$ : it depends on the other operating parameters. The difference between measured and calculated values is attributed to the fact that the drop does not spend enough time in the RZ to reach steady state.

The drop detaches when the overall restoring force reaches the maximum retaining capillary force

$$
\frac{\pi}{6} D_{d}^{3}\left(\left(\rho_{c}-\rho_{d}\right) g+\frac{1}{2} \rho_{d} \omega_{1}^{2} A_{d}^{\#}(\omega)\right)=\pi D_{p} \gamma
$$

where $A_{d}^{\#}(\omega)=\min \left(A_{d}(\omega), A_{d}^{\max }\right)$ since the drop does not spend enough time in the RZ to reach amplitudes exceeding $A_{d}^{\max }$. As an example, $D_{d}$ is calculated from eq. (9) for growing drops in the conditions of fig. 2(a), with $A_{d}^{\max }=4.5 \phi A_{p p}$ and $\beta$ from eq. (8). Results are shown by the solid line in fig. 2(a). The transition from dripping to stretching is well reproduced. The model from eq. (9) was applied with $A_{d}^{\max } /\left(\phi A_{p p}\right)$ estimated between $80 \mathrm{~Hz}$ and $100 \mathrm{~Hz}$ for each $D_{p}$ (for $A_{p p}<A_{t h}$ ). Results are shown in fig. 4 . The model is in good agreement with the experiments except for $D_{p}=0.11$ $\mathrm{mm}$. However, the discrepancy is coherent with the one observed for $D_{p}=0.11 \mathrm{~mm}$ in fig. 6 and is explained in the same manner.

Above $A_{t h}$, drop size decreases with increasing $A_{p p}$ because the detachment criterion is satisfied earlier in the RZ (for $\omega_{1}>\omega$ ). The model overestimates drop sizes above $A_{t h}$, attributed to nonlinear effects [8,9]. A downwards shift in resonance frequency occurs when increasing $A_{p p}$ for soft nonlinear oscillators [16]. Below $A_{t h}$, drop sizes are also overestimated: a resonance mode higher than mode 1 is excited and this is not taken into account in eq. (9).

We note that the present model does not account for the scaling $A_{t h} \sim \omega^{-1}$ observed in fig. 3 since eq. (9) under steady-state assumption leads to $A_{t h} \sim \omega^{-1 / 2}$. The LFHO assumption is too simplified to capture all the features of the transition. Also, the transient stage and nonlinear effects are not modeled.

Elongation-based analysis. - Similarly to Wilkes and Basaran [10], we also examined the maximum length reached by the drop prior to its detachment. At $A_{t h}$, we measured the maximum drop length $L_{\max }$ and defined the elongation ratio as the maximum drop length divided by the detached drop diameter. Dimensional analysis shows that $L_{\max } / D_{d}$ should essentially depend on the drop to pore diameter ratio $D_{d} / D_{p}$, since the physicochemical properties are set.

We report elongation ratio values as a function of $D_{d} / D_{p}$ (fig. 7) for all tested forcing frequencies and pore diameters. Although the statistical dispersion is significant, the larger the drops are compared to the pore, the lower the critical elongation ratio needed for detachment. On average, the critical elongation ratios decrease from about 1.4 to 1.1 when $D_{d} / D_{p}$ varies from 1.4 to 12 .

The decreasing trend can be explained by the results of Strani and Sabetta [6]. Indeed, they reported that when the drop to pore diameter ratio tends to infinity, the $n=1$ vibration mode degenerates into a zero-frequency rigid motion. Thus, for a large but finite $D_{d} / D_{p}$, the drop essentially experienced a rigid motion with the deformation mainly localised at the neck. This explains the lower drop elongation ratios for $D_{p}=0.11 \mathrm{~mm}$. 
For $D_{p}=0.75 \mathrm{~mm}$, critical elongation ratios are below the trend. It is attributed to the fact that, for $D_{p}=0.75 \mathrm{~mm}$, drop breakup occurred partly inside the tube rather than at the surface. Then, the measured method for $L_{\max }$ (from capillary tip to apex) underestimates the real drop length.

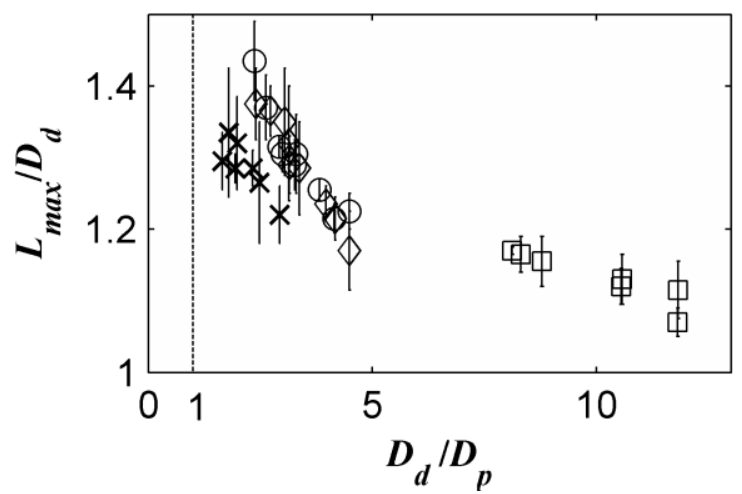

Figure 7: Elongation ratio function of drop to pore diameter ratio $(f=30 \mathrm{~Hz}-150 \mathrm{~Hz})$ : (口) $D_{p}=0.11 \mathrm{~mm}$, $(\diamond) D_{p}=0.32 \mathrm{~mm}$, (o) $D_{p}=0.35 \mathrm{~mm},\left(\right.$ x) $D_{p}=0.75 \mathrm{~mm}$.

The comparison with the simulation results of Wilkes and Basaran [10] are difficult since in their results, only a drop fraction is ejected. Moreover, the drop equivalent diameter is smaller than $D_{p}$. The closest case to our study is a $D_{d} / D_{p}$ value slightly lower than 1 . In this case, a critical elongation ratio of the order of 2 is reported, consistent with our results. According to their simulation snapshots, drop deformation creates pressure gradients inside the drop, leading to flow out of the neck, pinch-off and thus drop detachment. A similar scenario is expected in our experiments.

Conclusion. - Drop generation from a vibrating tube exhibits a transition from dripping to stretching mode when increasing the forcing amplitude at a set forcing frequency. In stretching mode, drops are significantly smaller from a threshold amplitude and above. During drop growth, the bound drop resonates in first mode once its resonance frequency and the forcing frequency coincide. It detaches by a so-called stretching mode if its elongation ratio exceeds a critical value, function of the drop to pore diameter ratio. The main features of this transition, i.e. threshold and drop diameter, can be described by a simple LFHO model using the expression of Bisch et al. for bound drop resonance frequency.

Acknowledgements. - We thank J. Trubuil and T. Martin for their help with designing and manufacturing the experimental setup.

\section{References}

[1] T. Nakashima, M. Shimizu and M. Kukizaki, «Membrane Emulsification by Microporous Glass », Key Engineering Materials, 61-62, p. 513-516, 1992.

[2] Holdich R. G., Dragosavac M. M., Vladisavljevic G. T. and Kosvintsev S. R., " Membrane emulsification with oscillating and stationary membranes », Ind. Eng. Chem. Res., 49 (8), p. 38103817, 2010.

[3] C. Arnaud, «Procédé et dispositif de fabrication d'une dispersion ou d'une émulsion », European patent EP 1551540 B1, 2006. 
[4] E. Lepercq-Bost, «Etude d'un procédé d'émulsification par membrane vibrante: compréhension des mécanismes, modélisation et optimisation », PhD Thesis, Ecole Centrale Paris, p. 224-233, 2008.

[5] C. Bisch, A. Lasek and H. Rodot, "Comportement hydrodynamique de volumes liquides sphériques semi-libres en apesanteur simulée », J. Méc. Théor. Appl., 1, p. 165-183, 1982.

[6] M. Strani M. and F. Sabetta, «Free vibrations of a drop in partial contact with a solid support », $J$. Fluid Mech., 141, p. 233-247, 1984.

[7] H. Lamb, Hydrodynamics, 6th Edition (Cambridge University Press, Cambridge, UK), p. 473-475, 1932.

[8] D. W. DePaoli, J. Q. Feng, O. A. Basaran, et T. C. Scott, «Hysteresis in forced oscillations of pendant drops », Phys. Fluids, 7, p. 1181-1183, 1995.

[9] E. D. Wilkes et O. A. Basaran, «Hysteretic response of supported drops during forced oscillations », J. Fluid Mech., 393, p. 333-356, 1999.

[10] E. D. Wilkes et O. A. Basaran, « Drop Ejection from an Oscillating Rod», J. Colloid Interface Sci., 242, p. 180-201, 2001.

[11] H.-Y. Kim, « Drop fall-off from the vibrating ceiling », Phys. Fluids, 16, p. 474-477, 2004.

[12] T. Tate, "On the magnitude of a drop of liquid formed under different circumstances », Phil. Mag., 27, p. 176-180, 1864.

[13] W. D. Harkins and F. E. Brown, « The determination of surface tension (free surface energy), and the weight of falling drops: the surface tension of water and benzene by the capillary height method », J. Am. Chem. Soc., 41, p. 499-524, 1919.

[14] Y. H. Mori, « Harkins-brown correction factor for drop formation », AIChE J., 36, p. 1272-1274, 1990.

[15] E. Butikov, Simulations of Oscillatory Systems (CRC Press, Taylor \& Francis Group), p. 38-45, 2015.

[16] E. D. Wilkes et O. A. Basaran, «Forced oscillations of pendant (sessile) drops », Phys. Fluids, 9, p. 1512-1528, 1997. 